\title{
Mid-aortic syndrome is associated with increased left ventricular mass index in Takayasu arteritis
}

\author{
Xu Meng^, Lin Zhao, Xueqi Dong, Xiongjing Jiang, Jun Cai, Huimin Zhang, Wenjun Ma, Haiying Wu, \\ Ying Lou, Linping Wang, Xianliang Zhou \\ Department of Cardiology, Fuwai Hospital, National Center for Cardiovascular Disease, Chinese Academy of Medical Sciences and Peking Union \\ Medical College, Beijing, China \\ Contributions: (I) Conception and design: X Meng, L Wang, Y Lou; (II) Administrative support: X Zhou, L Song; (III) Provision of study materials or \\ patients: X Jiang, J Cai, H Wu; (IV) Collection and assembly of data: L Zhao, X Dong; (V) Data analysis and interpretation: X Meng, H Zhang, W \\ Ma; (VI) Manuscript writing: All authors; (VII) Final approval of manuscript: All authors. \\ Correspondence to: Linping Wang. Department of Cardiology, Fuwai Hospital, National Center for Cardiovascular Disease, Chinese Academy of \\ Medical Sciences and Peking Union Medical College No. 167, Beilishi Road, Beijing 100037, China. Email: wanglping@139.com; Xianlaing Zhou. \\ Department of Cardiology, Fuwai Hospital, National Center for Cardiovascular Disease, Chinese Academy of Medical Sciences and Peking Union \\ Medical College No. 167, Beilishi Road, Beijing 100037, China. Email: zhouxianliang0326@hotmail.com.
}

Background: Mid-aortic syndrome (MAS) may induce changes in cardiac structure among patients with Takayasu arteritis (TA).

Methods: Consecutive adult patients with TA (January 1, 2011 to January 1, 2018) were enrolled and their data was retrospectively analyzed.

Results: Patients were divided into MAS group (100/457 patients, 21.8\%) and non-MAS group (357, $78.1 \%)$. The left ventricular mass index (LVMI) was higher in the MAS group than the non-MAS $\left(113.78 \pm 26.82\right.$ versus $100.74 \pm 23.66 \mathrm{~g} / \mathrm{m}^{2}$, respectively; $\left.\mathrm{P}<0.001\right)$. The MAS group showed higher prevalence than the non-MAS group of mild-to-severe mitral regurgitation $(9.0 \%$ and $3.9 \%$, respectively; $\mathrm{P}=0.040)$ and aortic regurgitation ( $26 \%$ and $14.8 \%$, respectively; $\mathrm{P}=0.003$ ). No difference was found in the rates of heart failure (27.0\% and $19.9 \%$ for MAS and non-MAS, respectively; $\mathrm{P}=0.126)$. The MAS group also showed lower estimated glomerular filtration rates than the non-MAS group $(89.93 \pm 18.89$ versus $96.16 \pm 21.60 \mathrm{~mL} / \mathrm{min}$ $/ 1.73 \mathrm{~m}^{2}$, respectively; $\mathrm{P}=0.009$ ) and higher prevalence of renal artery stenosis ( $57 \%$ versus $43.7 \% ; \mathrm{P}=0.018$ ). MAS was independently related to greater LVMI in both unadjusted model $[\beta=12.60 ; 95 \%$ confidence interval (CI): 7.09-18.11; $\mathrm{P}<0.001]$ and the model adjusted for multiple indices $(\beta=9.91 ; 95 \% \mathrm{CI}: 4.57-$ $15.25 ; \mathrm{P}<0.001)$ in multivariate linear analysis. The LVMI significantly decreased from $111.49 \pm 25.65$ to $100.36 \pm 22.91 \mathrm{~g} / \mathrm{m}^{2}(\mathrm{P}<0.001)$ among 55 patients who underwent successful revascularization treatment for MAS, while no significant difference $(\mathrm{P}=0.635)$ was observed among patients treated with medicine alone.

Conclusions: TA-induced MAS is a potential independent risk factor for increased LVMI, and revascularization therapy for MAS is effective in reversing structural changes in the heart.

Keywords: Takayasu arteritis (TA); mid-aortic syndrome (MAS); left ventricular mass index (LVMI); aortic surgery

Submitted Nov 16, 2020. Accepted for publication Mar 10, 2021.

doi: $10.21037 /$ atm-20-7508

View this article at: https://dx.doi.org/10.21037/atm-20-7508

$\wedge$ ORCID: 0000-0001-5829-5380. 


\section{Introduction}

Takayasu arteritis (TA) is a rare, idiopathic systemic vasculitis that mainly involves the aorta and its main branches, leading to multiple structural abnormalities such as wall thickening, fibrosis, stenosis, and even occlusion (1). TA-induced stenosis of the distal thoracic and/or abdominal aorta can induce mid-aortic-syndrome (MAS) (2), a clinical syndrome usually manifesting as upper-limb hypertension and lower-limb hypotension. TA is an extremely rare cause of secondary MAS; however, it is still noteworthy because almost all reported cases had concurrent organ damage $(2,3)$. The mortality in TA was reported to be nearly $10 \%$, and the 10 -year complication-free rate is no more than $50 \%$. Heart failure is considered the key cause of mortality among TA patients (4). Several sporadic cases of severe heart failure were reported in patients with TA-induced MAS, which is a rare but potential life-threatening condition $(3,5,6)$.

Our center has reported five pediatric patients with TAinduced MAS, manifesting as acute heart failure (3). Among those five, two patients died from cardiac shock and three patients recovered after treatment (3). However, except for those extremely severe cases, information about the general effects of TA-induced MAS on cardiac structure and function is still unavailable. Changes in heart structure commonly occur earlier than cardiac dysfunction, mainly presenting as ventricular wall thickening and dilation of the chambers. Left ventricular mass index (LVMI), as a sensitive index reflecting changes in cardiac structure, is thought to be associated with greater probability of progressing to cardiac dysfunction and higher prevalence of cardiovascular disease $(7,8)$. Therefore, we put forward the theory that TA-related MAS may induce structural abnormalities before the occurrence of detectable cardiac dysfunction, which may be reversed by effective treatments. This retrospective study summarizes clinical findings from 100 typical patients with TA-related MAS and compares those findings to a control group of 357 TA patients without MAS, to elucidate the influence of TArelated MAS on cardiac structure and function.

We present the following article in accordance with the STROBE reporting checklist (available at https://dx.doi. org/10.21037/atm-20-7508).

\section{Methods}

\section{Patients}

Consecutive adult patients diagnosed with TA, who were admitted to Fuwai Hospital from January 1, 2011 to
January 1 2018, were identified. TA was diagnosed based on the American College of Rheumatology's revised criteria (1990) (9). Diagnostic criteria for MAS were as follows: (I) segmental and/or focal narrowing $>50 \%$ of the normal diameter, or occlusive lesion of the distal thoracic and/ or abdominal aorta identified by at least one imaging examination; (II) supine arm-leg blood pressure gradient $>20 \mathrm{mmHg}(2,10)$. Patients younger than 18 years old at admission were excluded. All TA patients meeting the inclusion criteria of MAS were enrolled in the MAS group, whereas those without MAS were included in the non-MAS group.

This study was approved by the Ethics Committee of Fuwai Hospital (2020-1321), and complied with the Declaration of Helsinki (as revised in 2013) (World Medical Assembly) and its amendments. Informed consent was waived because our research retrospectively used deidentified data. Patients or the public were not involved in the design, or conduct, or reporting, or dissemination plans of our research.

\section{Data collection and imaging tests}

Information about demographic characteristics, clinical manifestations and imaging were retrospectively collected by two trained researchers. Hypertension was defined as a mean value of systolic blood pressure $>140 \mathrm{mmHg}$ and/ or diastolic blood pressure $>90 \mathrm{mmHg}$, evaluated in both upper limbs on at least two office readings. The reading of higher value was recorded among patients without involvement of the subclavian artery, whereas the reading from the normal side was used for patients with unilateral subclavian artery involvement. When both sides of the subclavian artery were involved, central blood pressure, directly measured from the root of the ascending aorta during angiography, was acquired (in 51 patients); blood pressure measurements were unavailable for patients who did not undergo angiography. Mean arterial pressure $=1 / 3$ $\times$ systolic blood pressure $+2 / 3 \times$ diastolic blood pressure.

Computed tomography angiography and/or magnetic resonance imaging were used as screening methods when clinical clues indicated an aortic narrowing (11). These images were independently evaluated by two experienced cardiologists in a blinded manner. We categorized the TA patients into five classifications based on Hata and Numano's criteria (12).

Transthoracic echocardiography was conducted in all subjects using the commercially available Philips IE33 
system (Philips Medical Systems, Eindhoven, Netherlands). Measures of ascending aorta diameter, left atrial diameter, interventricular septal end-diastolic thickness (IVST), left ventricular posterior wall end-diastolic thickness (LPWT), left ventricular end-diastolic dimension (LVDd) and left ventricular ejection fraction were extracted. Body surface area $=0.0061 \times$ body height $(\mathrm{cm})+0.0128 \times$ body weight $(\mathrm{kg})$ $-0.1529\left(\mathrm{~m}^{2}\right)$. Left ventricular mass $=0.8 \times 1.04 \times[($ IVST + $\left.\mathrm{LPWT}+\mathrm{LVDd})^{3}-\mathrm{LVDd}^{3}\right]+0.6 \mathrm{~g} . \mathrm{LVMI}=$ left ventricular mass /body surface area $\left(\mathrm{g} / \mathrm{m}^{2}\right)$.

The estimated glomerular filtration rate was calculated using the Chronic Kidney Disease Epidemiology Collaboration equation (2009) (13). Body mass index was calculated with weight $(\mathrm{kg}) / \mathrm{height}^{2}\left(\mathrm{~m}^{2}\right)$. Heart failure was diagnosed if a patient was classified as New York Heart Association functional class II-IV (14).

\section{Treatment}

Medication therapy was the basic treatment for all TA patients to maintain erythrocyte sedimentation rate and C-reactive protein at a normal range, mainly including prednisone and immunosuppressive agents. The indications for revascularization were an aortic diameter reduction of more than $70 \%$, accompanied by hypertension, lowerlimb claudication and/or left ventricular dysfunction. Aortography was performed by the same experienced physician in our center when revascularization treatment was being considered. The first-line treatment was percutaneous transluminal angioplasty; those who were not suitable for percutaneous transluminal angioplasty were treated with a surgical operation.

\section{Follow-up and outcomes}

Patients were asked to visit the clinic at 1, 3, 6 and 12 months after the discharge and every year thereafter. Data acquired from the last follow-up were analyzed to evaluate the effect of revascularization therapy on blood pressure, heart and renal. Clinical outcomes, including vascular complications and all-cause death, were also investigated during follow-up. Vascular complications were defined as new-onset of more than one of the following events: myocardial infarction, heart failure, stroke and endstage renal failure. The follow-up period was counted from the date of discharge to the date of latest follow-up.

\section{Data analysis}

Data were analyzed using Statistical Package for the Sciences software (Version 23.1, IBM Corp., Armonk, NY, USA). Homogeneity of variance was examined with the Levene test (two-sided $\mathrm{P}>0.05$ ). Continuous variables were expressed as mean \pm standard deviation, and categorical variables were expressed as numbers and percentages. Continuous independent variables were compared with independent $t$-tests. Paired $t$-tests were used to compare continuous variables between baseline and the latest followup in the same patient. Categorical variables were compared with a chi-square test or Fisher exact test.

Multivariate linear analysis was conducted to investigate the correlation of MAS and LVMI. Bivariate correlation between the LVMI and independent variables was judged by Pearson or Kendall's tau-b correlation coefficient. Dichotomous variables were recoded as " 0 " and " 1 " when tested. For example, we recoded sex into "1" for male and " 0 " for female. In multivariate linear analysis, we included the independent parameters with $\mathrm{P}<0.10$ in the bivariate correlation analysis as covariates of MAS. Items that correlate with cardiac hypertrophic remodeling in our clinical experience and previous studies were also included as covariates. The stepwise forward selection method was used in the multivariate linear analysis. The entering and removal levels were 0.05 and 0.10 , respectively. Two-sided $\mathrm{P}<0.05$ was considered statistically significant.

\section{Results}

\section{Characteristics of subjects}

From 1 January, 2011 to 1 January, 2018, a total of 457 adult patients were diagnosed with TA, including 100 (21.9\%) with MAS (MAS group) and 357 (78.1\%) without MAS (non-MAS group). Basic clinical characteristics of the MAS and non-MAS groups are summarized in Table 1. Compared with the non-MAS group, both hypertension (70.0\% MAS vs. $56.0 \%$ non-MAS; $\mathrm{P}=0.012$ ) and resistant hypertension $(23.0 \%$ vs. $13.4 \% ; \mathrm{P}=0.020)$, as well as the mean values of systolic blood pressure $(155.81 \pm 29.12 v s$. $150.24 \pm 22.90 \mathrm{mmHg} ; \mathrm{P}=0.045)$ and mean arterial pressure $(105.76 \pm 16.39$ vs. $101.76 \pm 16.24 \mathrm{mmHg} ; \mathrm{P}=0.031)$ were significantly higher in the MAS group. In the MAS group, type $\mathrm{V}(67 / 100,67.0 \%)$ was the most common type of TA, followed by type I (17/100, 17.0\%). In the non-MAS group, 
Table 1 Medical records of the patients in the MAS group and NMAS group at baseline

\begin{tabular}{|c|c|c|c|}
\hline Characteristics & MAS group $(n=100)$ & NMAS group $(\mathrm{n}=357)$ & $P$ value \\
\hline Male (\%) & $17(17.0)$ & $52(14.6)$ & 0.548 \\
\hline Disease duration, years & $9.43 \pm 6.93$ & $9.22 \pm 7.35$ & 0.797 \\
\hline Body mass index, $\mathrm{kg} / \mathrm{m}^{2}$ & $23.29 \pm 3.87$ & $22.92 \pm 3.31$ & 0.350 \\
\hline Systolic blood pressure, $\mathrm{mmHg}$ & $155.81 \pm 29.12$ & $150.24 \pm 22.90$ & 0.045 \\
\hline Diastolic blood pressure, $\mathrm{mmHg}$ & $80.74 \pm 15.35$ & $77.53 \pm 16.79$ & 0.086 \\
\hline Mean arterial pressure, $\mathrm{mmHg}$ & $105.76 \pm 16.39$ & $101.76 \pm 16.24$ & 0.031 \\
\hline Smoking (\%) & $9(9.0)$ & $26(7.3)$ & 0.568 \\
\hline Dyslipidemia (\%) & $28(28.0)$ & $96(26.9)$ & 0.825 \\
\hline Diabetes mellitus (\%) & $6(6.0)$ & $15(4.2)$ & 0.448 \\
\hline Total cholesterol, mmol/L & $4.30 \pm 1.0$ & $4.42 \pm 1.11$ & 0.326 \\
\hline LDLC, mmol/L & $2.58 \pm 0.93$ & $2.57 \pm 0.88$ & 0.889 \\
\hline Triglyceride, $\mathrm{mmol} / \mathrm{L}$ & $1.28 \pm 0.53$ & $1.33 \pm 0.78$ & 0.456 \\
\hline $\mathrm{HbA1c}$ & $5.63 \pm 0.76$ & $5.56 \pm 0.73$ & 0.401 \\
\hline Creatine, $\mu \mathrm{mol} / \mathrm{L}$ & $74.83 \pm 20.50$ & $70.14 \pm 18.39$ & 0.029 \\
\hline $\mathrm{ESR}, \mathrm{mm} / \mathrm{h}$ & $16.09 \pm 16.51$ & $17.16 \pm 19.35$ & 0.616 \\
\hline III & $12(12.0)$ & $52(14.6)$ & 0.513 \\
\hline IV & $17(17.0)$ & $31(8.7)$ & 0.017 \\
\hline V & $67(67.0)$ & $116(32.5)$ & $<0.001$ \\
\hline C & $16(16.0)$ & $56(15.7)$ & 0.939 \\
\hline$P$ & $16(16.0)$ & 69 (19.3) & 0.450 \\
\hline
\end{tabular}

Values are the mean \pm standard deviation, or number (\%). *Takayasu arteritis cases were classified based on Hata and Numano's criteria: type I: involvement of the aortic arch and its primary branches; type lla: involvement of the ascending aorta, aortic arch and its branches; type IIb: involvement of ascending aorta, aortic arch with its branches, and thoracic descending aorta; type III: involvement of thoracic descending aorta, abdominal aorta; type IV: involvement of the abdominal aorta; type V: type Ila combined with type IV; C: involvement of coronary artery; P: involvement of pulmonary artery. eGFR, estimated glomerular filtration rate; ESR: erythrocyte sedimentation rate; HbA1c, glycosylated hemoglobin; hsCRP, high sensitivity C reactive protein; LDLC, low density lipoprotein cholesterol; MAS, mid-aortic syndrome; NMAS, non-mid-aortic syndrome. 
Table 2 Evaluation of organ damage in the MAS group and NMAS group

\begin{tabular}{|c|c|c|c|}
\hline Variables & MAS group $(n=100)$ & NMAS group $(n=357)$ & $P$ value \\
\hline \multicolumn{4}{|l|}{ Heart } \\
\hline $\mathrm{AAD}, \mathrm{mm}$ & $3.79 \pm 3.61$ & $32.48 \pm 3.52$ & 0.001 \\
\hline $\mathrm{LAD}, \mathrm{mm}$ & $35.16 \pm 5.06$ & $33.32 \pm 4.94$ & 0.001 \\
\hline LVDD, mm & $47.75 \pm 4.02$ & $46.41 \pm 3.22$ & 0.001 \\
\hline DPWT, mm & $10.21 \pm 1.42$ & $9.66 \pm 1.37$ & $<0.001$ \\
\hline LVMI, $g / \mathrm{m}^{2}$ & $113.78 \pm 26.82$ & $100.74 \pm 23.66$ & $<0.001$ \\
\hline Mild to severe MR (\%) & $9(9.0)$ & $14(3.9)$ & 0.040 \\
\hline Mild to severe AR (\%) & $26(26.0)$ & $53(14.8)$ & 0.003 \\
\hline CA involvement (\%) & $16(16.0)$ & $56(15.7)$ & 0.939 \\
\hline MI (\%) & $3(3.0)$ & $16(4.5)$ & 0.777 \\
\hline \multicolumn{4}{|l|}{ Renal } \\
\hline Creatine, $\mu \mathrm{mol} / \mathrm{L}$ & $74.83 \pm 20.50$ & $70.14 \pm 18.39$ & 0.029 \\
\hline eGFR, mL/min/1.73 m² & $89.93 \pm 18.89$ & $96.16 \pm 21.60$ & 0.009 \\
\hline RAS (\%) & $57(57.0)$ & $156(43.7)$ & 0.018 \\
\hline Kidney shrinkage (\%) & $12(12.0)$ & $21(5.9)$ & 0.038 \\
\hline \multicolumn{4}{|l|}{ Nervous system (\%) } \\
\hline
\end{tabular}

Values are the mean \pm standard deviation or number (\%). AAD, ascending aorta diameter; AR, aortic regurgitation; CA, coronary artery; DPWT, diastolic posterior wall thickness; eGFR, estimated glomerular filtration rate; HF, heart failure; IVST, interventricular septum thickness; LAD, left atrial diameter; LVDD, left ventricular end diastolic diameter; LVEF, left ventricular ejection fraction; LVMI, left ventricular mass index; MAS, mid-aortic syndrome; MI, myocardial infarction; MR, mitral regurgitation; NMAS, non-mid-aortic syndrome; RAS: renal artery stenosis.

the most frequent types were type I $(117 / 357,32.8 \%)$ and type $\mathrm{V}(116 / 357,32.5 \%)$.

\section{Organ damage}

Heart, renal, nervous system and fundus changes in the MAS and non-MAS groups are compared in Table 2. In the MAS group, the ascending aorta diameter, left atrial diameter, LVDd, IVST and LVMI were larger than those in the non-MAS group. The prevalence of mild-to-severe mitral regurgitation and aortic regurgitation were also higher in the
MAS group. As for renal function, the mean level of estimated glomerular filtration rate was significantly lower, and the prevalence of renal artery stenosis and kidney shrinkage were higher in the MAS group than the non-MAS group.

\section{Potential risk factors inducing cardiac structure damage}

Table 3 shows the bivariate correlations between the LVMI and the independent variables. Judged by Pearson or Kendall's tau-b correlation coefficients, indexes with $\mathrm{P}<0.1$ were included as the covariates of MAS in the stepwise 
Table 3 Variables tested for inclusion in the multivariate linear regression analysis for the LVMI

\begin{tabular}{|c|c|c|}
\hline Variables & Correlation coefficient $(95 \% \mathrm{Cl})^{8}$ & $P$ value \\
\hline Male & 0.084 (0.006 to 0.163$)$ & $0.028^{\#}$ \\
\hline Disease duration, years & $0.094(-0.002$ to 0.194$)$ & $0.056^{\#}$ \\
\hline Body mass index, $\mathrm{kg} / \mathrm{m}^{2}$ & $-0.103(-0.198$ to -0.010$)$ & $0.037^{\#}$ \\
\hline Smoking & $0.052(-0.02$ to 0.040$)$ & 0.170 \\
\hline Total cholesterol, mmol/L & $-0.025(-0.114$ to 0.069$)$ & 0.612 \\
\hline Triglyceride, $\mathrm{mmol} / \mathrm{L}$ & $0.018(-0.060$ to 0.112$)$ & 0.720 \\
\hline LDLC, mmol/L & $-0.013(-0.107$ to 0.072$)$ & 0.798 \\
\hline hsCRP, mg/L & $0.153(0.054$ to 0.254$)$ & $0.002^{\#}$ \\
\hline eGFR, mL/min/1.73 $\mathrm{m}^{2}$ & $-0.165(-0.261$ to -0.076$)$ & $0.001^{\#}$ \\
\hline Mid-aortic syndrome & 0.158 (0.080 to 0.233$)$ & $<0.001^{\#}$ \\
\hline \multicolumn{3}{|l|}{ Heart } \\
\hline LVEF, \% & $-0.139(-0.246$ to -0.030$)$ & $0.005^{\#}$ \\
\hline Mild to severe MR & $0.094(0.028$ to 0.156$)$ & $0.014^{\#}$ \\
\hline Mild to severe AR & $0.142(0.072$ to 0.218$)$ & $<0.001^{\#}$ \\
\hline CA involvement & $-0.008(-0.079$ to 0.058$)$ & 0.831 \\
\hline
\end{tabular}

*Values are the mean \pm standard deviation or number (\%). ${ }^{\&}$ Bivariate correlations between two continuous variables were judged with Pearson correlation coefficient, and those between one continuous variable and one dichotomous variable were judged with Kendall's tau-b correlation coefficient. "The variables with a $\mathrm{P}$ value were included in the multivariate linear analysis. LVMI, left ventricular mass index; AR, aortic regurgitation; CA, coronary artery; Cl, confidence interval; eGFR, estimated glomerular filtration rate; ESR, erythrocyte sedimentation rate; HbA1c, hemoglobin A1c; hsCRP, high sensitivity C reactive protein; LDLC, low density lipoprotein cholesterol; LVEF, left ventricular ejection fraction; MI, myocardial infarction; MR, mitral regurgitation.

forward selection method of multivariate linear analysis. Table 4 shows the relationships between the MAS and the LVMI. In unadjusted analyses, the MAS was significantly associated with greater LVMI ( $\beta=12.60$; 95\% CI: 7.0918.11; $\mathrm{P}<0.001)$. In multivariate analyses accounting for age and sex $(\beta=12.89 ; 95 \%$ CI: $7.49-18.30 ; \mathrm{P}<0.001)$, as well as in the model selected by the stepwise forward method in multivariate linear analysis, adjusted for mild-to-severe aortic regurgitation, body mass index, estimated glomerular filtration rate, left ventricular ejection fraction, erythrocyte sedimentation rate and high sensitivity $\mathrm{C}$ reactive protein ( $\beta=9.91 ; 95 \%$ CI: 4.57 to $15.25 ; \mathrm{P}<0.001$ ), MAS was again found to be significantly associated with higher LVMI.

\section{Treatment and follow-up of patients with TA-induced $M A S$}

All patients in the MAS group were treated with prednisone, starting with a daily dose of $0.5 \mathrm{mg} / \mathrm{kg}$ for 1 month and tapering to a maintenance dosage of $5-10 \mathrm{mg} /$ day, to suppress inflammatory reactions. Antihypertensive medicines were adjusted to keep blood pressure $<140 / 90 \mathrm{mmHg}$ among patients with hypertension. A total of 63 patients with MAS $>70 \%$ underwent aortography during their hospitalization. Fifty-five of them underwent successful aortic stenting. Guide wire failed to progress through the occlusive aortic lesion during 
Table 4 Association between mid-aortic syndrome and left ventricular mass index (LVMI) among patients with Takayasu arteritis

\begin{tabular}{|c|c|c|c|c|}
\hline Model & Variables & $\beta(95 \% \mathrm{Cl})$ & $P$ value & Adj. $R^{2}$ \\
\hline 2 & Demographic factors & 12.89 (7.49 to 18.30$)$ & $<0.001$ & 0.051 \\
\hline 3 & Selected by stepwise forward method & 9.91 (4.57 to 15.25$)$ & $<0.001$ & 0.145 \\
\hline
\end{tabular}

*Model 1, unadjusted; model 2, adjusted for demographic characteristics including age and sex; model 3, the best model selected by the stepwise forward method, adjusted for mild-to-severe aortic regurgitation, body mass index, estimated glomerular filtration rate, left ventricular ejection fraction, erythrocyte sedimentation rate ESR and high sensitivity $\mathrm{C}$ reactive protein. $\mathrm{Cl}$, confidence interval; Adj. $\mathrm{R}^{2}$, adjusted $R^{2}$.

Table 5 Comparison of blood pressure, and cardiac structure and function between baseline and follow-up

\begin{tabular}{|c|c|c|c|c|c|c|}
\hline Variables & \multicolumn{3}{|c|}{ Medicine with revascularization $(n=55)$} & \multicolumn{3}{|c|}{ Medicine $(n=30)$} \\
\hline \multicolumn{7}{|l|}{ Blood pressure } \\
\hline $\mathrm{SBP}, \mathrm{mmHg}$ & $157.56 \pm 25.7$ & $137.78 \pm 14.63$ & $<0.001$ & $151.00 \pm 31.06$ & $139.97 \pm 17.89$ & 0.001 \\
\hline $\mathrm{DBP}, \mathrm{mmHg}$ & $82.25 \pm 17.94$ & $74.29 \pm 11.42$ & $<0.001$ & $77.50 \pm 12.23$ & $73.50 \pm 8.62$ & 0.008 \\
\hline \multicolumn{7}{|l|}{ Heart } \\
\hline $\mathrm{AAD}, \mathrm{mm}$ & $33.26 \pm 3.11$ & $31.26 \pm 4.32$ & $<0.001$ & $33.90 \pm 3.87$ & $33.83 \pm 3.81$ & 0.794 \\
\hline $\mathrm{LAD}, \mathrm{mm}$ & $34.49 \pm 5.05$ & $33.82 \pm 4.50$ & 0.058 & $35.87 \pm 5.12$ & $36.06 \pm 4.90$ & 0.501 \\
\hline LVDD, mm & $47.13 \pm 3.09$ & $46.53 \pm 2.92$ & 0.014 & $48.27 \pm 5.12$ & $47.87 \pm 5.43$ & 0.369 \\
\hline LVMI, $g / \mathrm{m}^{2}$ & $111.49 \pm 25.65$ & $100.36 \pm 22.91$ & $<0.001$ & $113.54 \pm 26.84$ & $112.44 \pm 27.99$ & 0.635 \\
\hline LVEF, \% & $62.32 \pm 11.62$ & $63.04 \pm 8.70$ & 0.218 & $62.44 \pm 8.65$ & $61.63 \pm 9.33$ & 0.058 \\
\hline \multicolumn{7}{|l|}{ Renal } \\
\hline Creatine, $\mu \mathrm{mol} / \mathrm{L}$ & $75.06 \pm 20.50$ & $72.16 \pm 17.85$ & 0.001 & $73.93 \pm 19.62$ & $74.47 \pm 20.70$ & 0.327 \\
\hline eGFR, mL/min/1.73 $\mathrm{m}^{2}$ & $90.45 \pm 20.04$ & $93.61 \pm 18.57$ & 0.002 & $89.20 \pm 18.69$ & $88.88 \pm 19.12$ & 0.714 \\
\hline
\end{tabular}

Values are the mean \pm standard deviation. AAD, ascending aorta diameter; DBP, diastolic blood pressure; eGFR, estimated glomerular filtration rate; IVST, interventricular septum thickness; LAD, left atrial diameter; LVDD, left ventricular end diastolic diameter; LVEF, left ventricular ejection fraction; LVMI, left ventricular mass index; MAP, mean arterial pressure; SBP, systolic blood pressure.

aortography in two patients. Both of them were successfully treated with extra-anatomic bypass surgery. Among the six patients who were not treated with revascularization therapy, there were five patients with aortic stenosis less than $70 \%$, and one patient with an abdominal aortic aneurysm near the stenotic lesion, so interventional therapy was refused because of surgical risk. Finally, 43 patients received medicine treatment only, including 36 patients with CT-identified aorta stenosis less than $70 \%, 4$ patients with contraindications for surgery, and 3 patients who refused the operation because of the risk of the operation.

Table 5 shows the comparisons of blood pressure, heart structure and function, and renal function between baseline and the latest follow-up among those patients treated with both medicine and revascularization, and those patients treated with medicine only. Clinical follow-up information was acquired for 55 of the 57 patients treated with both medicine and revascularization (53 patients underwent 
Table 6 Long-term clinical outcomes between the MAS group and the NMAS group

\begin{tabular}{llll}
\hline Variables & $\begin{array}{l}\text { MAS group } \\
(\mathrm{n}=88)\end{array}$ & $\begin{array}{l}\text { NMAS group } \\
(\mathrm{n}=323)\end{array}$ & P value \\
\hline Vascular complications & $18(20.5 \%)$ & $50(15.5 \%)$ & 0.266 \\
Myocardial infarction & $2(2.3 \%)$ & $8(2.5 \%)$ & 0.779 \\
Heart failure & $7(8.0 \%)$ & $11(3.4 \%)$ & 0.065 \\
Ischemic stroke & $7(8.0 \%)$ & $25(7.7 \%)$ & 0.947 \\
Hemorrhagic stroke & $0(0 \%)$ & $1(0.03 \%)$ & 0.485 \\
End-stage renal failure & $2(2.3 \%)$ & $5(1.5 \%)$ & 0.999 \\
All-cause death & $7(8.0 \%)$ & $18(5.6 \%)$ & 0.407 \\
\hline
\end{tabular}

aortic stenting and 2 patients underwent surgery), and 30 of the 43 patients treated with only medicine, with followup periods of $4.40 \pm 1.84$ years (range from 1.17 to 8.17 ) and $3.40 \pm 1.74$ years (range from 1.00 to 6.67 ), respectively. In patients treated with both medicine and revascularization, there were significant decreases in systolic blood pressure, diastolic blood pressure and mean arterial pressure, as well as the ascending aorta diameter, left atrial diameter, LVDd, LVST, DPWT, LVMI and plasma creatine, and increases in estimated glomerular filtration rate observed at the latest follow-up visit, compared with baseline. However, there were only significant decreases in blood pressure levels at the latest follow-up among patients treated with medicine alone, with no statistical differences identified for the heart and renal indices.

\section{Long-term clinical outcomes in the MAS group and the NMAS group}

Table 6 shows comparison of long-term clinical outcomes between the MAS group and the NMAS group. Follow-up data was acquired for 88 of 100 patients in the MAS group and 323 of 357 patients in the NMAS group, with followup periods of $4.02 \pm 1.83$ years (range from 1.00 to 8.17 ) and $4.25 \pm 1.29$ years (range from 0.75 to 9.25 ), respectively $(\mathrm{P}=0.276)$. No difference was found in the incidence of the vascular complications $(20.5 \%$ vs. $15.5 \% ; \mathrm{P}=0.266)$ and allcause death $(8.0 \%$ vs. $5.6 \% ; \mathrm{P}=0.407)$ between the MAS group and the NMAS group. In the MAS group, causes of death were heart failure in 5 , acute myocardial infarction in 1 , and sudden cardiac death in 1 patient. Among those who had NMAS, causes of death included heart failure in 9 , acute myocardial infarction in 3 , ischemic stroke in 3 , hemorrhagic stroke in 1 , sudden cardiac death in 2 patients.

\section{Discussion}

In this research, we first reported a cohort of adult TA patients with MAS, and further analyzed the organ damage, the relationship between MAS and the LVMI, the therapeutic effect of revascularization of MAS on blood pressure, heart and renal function and long-term clinical outcomes. The most-notable conclusions from this study are as follows: (I) more than one-fifth of TA patients had MAS; (II) patients with TA-induced MAS showed more changes in cardiac structure and severely damaged renal function; (III) TA-induced MAS was an independent risk factor inducing increased LVMI; (IV) revascularization therapy for MAS was effective in controlling blood pressure, reversing structural changes in the heart and improving renal function.

MAS can be caused by congenital or acquired etiologies (15). Previously, congenital disease was considered to be the main etiology of MAS $(16,17)$. Among adults, only small cohorts of patients with MAS induced by TA have been reported, mostly to investigate revascularization in MAS $(18,19)$. MAS typically features stenotic lesions on the distal thoracic and/or abdominal aorta, causing elevated blood pressure ahead of the lesion, and hypotension and ischemia beyond the lesion. In this research, we found that $21 \%(100 / 475)$ of TA patients had concurrent MAS, which has not been reported previously, and $70 \%$ of patients with TA-induced MAS had hypertension. Patients with MAS had significantly higher blood pressure than those without MAS did. Among TA patients, common mechanisms of hypertension include renal artery stenosis, stenosis of the thoracic descending aorta and abdominal aorta, and severe aortic regurgitation (20). That is consistent with the fact that all of the patients in our MAS group had coexisting stenotic lesions on the distal thoracic descending aorta and/ or abdominal aorta, along with elevated prevalence of renal artery stenosis and aortic regurgitation. In this research, the most common type of TA was type $\mathrm{V}(\mathrm{n}=183,40.0 \%)$ among the total cohort, and $36.6 \%$ of type- $\mathrm{V}$ patients had MAS. TA-induced MAS mostly occurred among TA patients with type $\mathrm{V}$ or type IV, both classifications involving the distal thoracic and/or abdominal aorta.

Organ damage is not rare among patients with TA $(1,3,21)$. Congestive heart failure, as the end-stage of heart damage, indicates poor prognoses, including CVD, and even death (7). Prevalence of heart failure was reported 
to range from $2.5 \%$ to $26.7 \%$ in different TA cohorts $(4,22,23)$, which is consistent with $21.4 \%(98 / 457)$ in our cohort. Traditional causes of heart failure in TA patients include elevated afterload, due to aortic regurgitation and hypertension. Myocarditis, accelerated atherosclerosis and pulmonary hypertension are also reportedly factors $(24,25)$. In this research, the proportion of patients with aortic regurgitation and hypertension, as well as blood pressure level, were higher in the MAS group than the non-MAS group. However, the rate of coexisting heart failure showed no significant difference between the MAS and non-MAS groups, perhaps because of the relatively short followup period. Despite this, we observed more-severe cardiac structural changes in the MAS group, especially in terms of higher LVMI. The LVMI, a sensitive index reflecting left ventricular hypertrophy, indicates hypertensionrelated target organ damage, with elevated risk for adverse cardiovascular outcomes (26). Increased LVMI is a compensatory mechanism in response to over-afterload of the left ventricle. In this research, we first found that MAS was an independent risk factor for elevated LVMI. There are several potential mechanisms. First, MAS-induced hypertension and AR will further increase the elevated afterload on the left ventricle. Second, renal ischemia, related to renal artery stenosis and/or severe aortic stenosis before the ostia of the renal artery, may stimulate secretion of renin and activate the renin-angiotensin-aldosterone system. Activation of the renin-angiotensin-aldosterone system may induce hypertrophy and hyperplasia in cardiac myocytes, and lead to perivascular and interstitial fibrosis, as well as stimulating extracellular collagen synthesis and interfering with collagen degradation $(27,28)$. Third, estimated glomerular filtration rate was significantly lower in the MAS group than in the non-MAS group, which may be associated with renal ischemia and hypertension-related renal damage. Renal dysfunction may also induce cardiac hypertrophy, left ventricle fibrosis, enlargement of myocytes and cardiac fibrosis (29).

A previous study showed that stenting was effective in controlling blood pressure and improving heart function in patients with TA-induced MAS (18). They also found a trend to reduction of the interventricular septum thickness with stenting, decreasing from $10.5 \pm 2.5$ to $9.7 \pm 1.9 \mathrm{~mm}$ ( $\mathrm{P}=0.07)$. Kim et al. observed that the LVMI was reduced from $164.2 \pm 65.8$ to $111.9 \pm 35.0 \mathrm{~g} / \mathrm{m}^{2}$ among 7 patients with TA-induced MAS after extra-anatomic bypass surgery; however, this improvement was not statistically significant $(\mathrm{P}=0.116)$ because of the extremely small sample size (19). In the present research, we observed significantly reduced LVMI among those who underwent successful revascularization therapy, which indicates reversion of the hypertrophic cardiac remodeling. That may provide benefits, from reduced blood pressure level and improvement of renal function, which were also observed in this research. Although we observed no difference in the incidence of the vascular complications and all cause death between the MAS group and the NMAS group, that may because of short follow-up period in the present research. Considering the recognized association between elevated LVMI and CVD, timely revascularization treatment of patients with TAinduced MAS may reduce the risk of CVD.

The main limitation of this research was that the retrospective and single-center design of the study inevitably induces selection bias. Furthermore, the retrospective nature made it impossible to determine a causal link between MAS and the elevated LVMI. However, the significant decrease in LVMI during long-term followup among TA-induced MAS patients who underwent successful revascularization treatment indicates that MAS may be a risk factor inducing elevated LVMI.

\section{Conclusions}

MAS is common in TA patients, involving more than one-fifth of them in our cohort. MAS was associated with elevated blood pressure, cardiac hypertrophic remodeling and renal damage among TA patients. TA-induced MAS may be an independent risk factor for increased LVMI. Revascularization therapy in patients with MAS was effective in reversing hypertrophic structural changes in the heart, controlling blood pressure, and improving renal function.

\section{Acknowledgments}

This work was supported by the National Key Research and Development Plan of China (grant number 2016YFC1300100), the CAMS Innovation Fund for Medical Science (grant number 2016-I2M-1-002) and the Central Research Institute Fund of Chinese Academy of Medical Sciences (grant number 2019XK320035). We thank Claire Barnes, PhD, from Liwen Bianji, Edanz Editing China (www.liwenbianji.cn/ac), for editing the English text of a draft of this manuscript. 


\section{Footnote}

Reporting Checklist: The authors have completed the STROBE reporting checklist. Available at https://dx.doi. org/10.21037/atm-20-7508

Data Sharing Statement: Available at https://dx.doi. org/10.21037/atm-20-7508

Conflicts of Interest: All authors have completed the ICMJE uniform disclosure form (available at https://dx.doi. org/10.21037/atm-20-7508). The authors have no conflicts of interest to declare.

Ethical Statement: The authors are accountable for all aspects of the work in ensuring that questions related to the accuracy or integrity of any part of the work are appropriately investigated and resolved. This study was approved by the Ethics Committee of Fuwai Hospital (2020-1321), and complied with the Declaration of Helsinki (as revised in 2013) (World Medical Assembly) and its amendments. Informed consent was waived because our research retrospectively used de-identified data.

Open Access Statement: This is an Open Access article distributed in accordance with the Creative Commons Attribution-NonCommercial-NoDerivs 4.0 International License (CC BY-NC-ND 4.0), which permits the noncommercial replication and distribution of the article with the strict proviso that no changes or edits are made and the original work is properly cited (including links to both the formal publication through the relevant DOI and the license). See: https://creativecommons.org/licenses/by-nc-nd/4.0/.

\section{References}

1. Alibaz-Oner F, Direskeneli H. Update on Takayasu's arteritis. Presse Med 2015;44:e259-65.

2. Lin YJ, Hwang B, Lee PC, et al. Mid-aortic syndrome: a case report and review of the literature. Int J Cardiol 2008;123:348-52.

3. Fan L, Zhang H, Cai J, et al. Middle aortic syndrome because of pediatric Takayasu arteritis admitted as acute heart failure: clinical course and therapeutic strategies. J Hypertens 2018;36:2118-9.

4. Comarmond C, Biard L, Lambert M, et al. Long-Term Outcomes and Prognostic Factors of Complications in Takayasu Arteritis: A Multicenter Study of 318 Patients.
Circulation 2017;136:1114-22.

5. Cheng KCA, Li YL. Mid-aortic syndrome secondary to Takayasu's disease. BMJ Case Rep 2017;2017.

6. Zaki SA. Middle aortic syndrome caused by Takayasu arteritis. Heart Views 2012;13:116-7.

7. Tsao CW, Gona PN, Salton CJ, et al. Left Ventricular Structure and Risk of Cardiovascular Events: A Framingham Heart Study Cardiac Magnetic Resonance Study. J Am Heart Assoc 2015;4:e002188.

8. Gottdiener JS, Arnold AM, Aurigemma GP, et al. Predictors of congestive heart failure in the elderly: the Cardiovascular Health Study. J Am Coll Cardiol 2000;35:1628-37.

9. Arend WP, Michel BA, Bloch DA, et al. The American College of Rheumatology 1990 criteria for the classification of Takayasu arteritis. Arthritis Rheum 1990;33:1129-34.

10. Dijkema EJ, Leiner T, Grotenhuis HB. Diagnosis, imaging and clinical management of aortic coarctation. Heart 2017;103:1148-55.

11. Erbel R, Aboyans V, Boileau C, et al. 2014 ESC Guidelines on the diagnosis and treatment of aortic diseases:

Document covering acute and chronic aortic diseases of the thoracic and abdominal aorta of the adult. The Task Force for the Diagnosis and Treatment of Aortic Diseases of the European Society of Cardiology (ESC). Eur Heart J 2014;35:2873-926.

12. Hata A, Noda M, Moriwaki R, et al. Angiographic findings of Takayasu arteritis: new classification. Int J Cardiol 1996;54 Suppl:S155-63.

13. Levey AS, Stevens LA, Schmid CH, et al. A new equation to estimate glomerular filtration rate. Ann Intern Med 2009;150:604-12.

14. Ponikowski P, Voors AA, Anker SD, et al. 2016 ESC Guidelines for the diagnosis and treatment of acute and chronic heart failure: The Task Force for the diagnosis and treatment of acute and chronic heart failure of the European Society of Cardiology (ESC)Developed with the special contribution of the Heart Failure Association (HFA) of the ESC. Eur Heart J 2016;37:2129-200.

15. Graham LM, Zelenock GB, Erlandson EE, et al. Abdominal aortic coarctation and segmental hypoplasia. Surgery 1979;86:519-29.

16. ten Dam K, van der Palen RL, Tanke RB, et al. Clinical recognition of mid-aortic syndrome in children. Eur J Pediatr 2013;172:413-6.

17. Kadoya Y, Zen K, Saburi M, et al. Mid-aortic Syndrome: A Rare Cause of Juvenile Hypertension. Intern Med 
2018;57:447.

18. Che W, Xiong H, Jiang X, et al. Stenting for middle aortic syndrome caused by Takayasu arteritis-immediate and long-term outcomes. Catheter Cardiovasc Interv 2018;91:623-31.

19. Kim HJ, Choi JW, Hwang HY, et al. Extra-Anatomic Ascending Aorta to Abdominal Aorta Bypass in Takayasu Arteritis Patients with Mid-Aortic Syndrome. Korean J Thorac Cardiovasc Surg 2017;50:270-4.

20. Qi Y, Yang L, Zhang H, et al. The presentation and management of hypertension in a large cohort of Takayasu arteritis. Clin Rheumatol 2018;37:2781-8.

21. Ritter JC, Ghosh J, Butterfield JS, et al. Chimney stent technique for treatment of severe abdominal aortic atherosclerotic stenosis. J Vasc Interv Radiol 2011;22:391-4.

22. Lee GY, Jang SY, Ko SM, et al. Cardiovascular manifestations of Takayasu arteritis and their relationship to the disease activity: analysis of 204 Korean patients at a single center. Int J Cardiol 2012;159:14-20.

23. Yang L, Zhang H, Jiang X, et al. Clinical manifestations

Cite this article as: Meng X, Zhao L, Dong X, Jiang X, Cai J, Zhang H, Ma W, Wu H, Lou Y, Wang L, Zhou X. Mid-aortic syndrome is associated with increased left ventricular mass index in Takayasu arteritis. Ann Transl Med 2021;9(14):1124. doi: 10.21037/atm-20-7508 and longterm outcome for patients with Takayasu arteritis in China. J Rheumatol 2014;41:2439-46.

24. An X, Han Y, Zhang B, et al. Takayasu arteritis presented with acute heart failure: case report and review of literature. ESC Heart Fail 2017;4:649-54.

25. Talwar KK, Kumar K, Chopra P, et al. Cardiac involvement in nonspecific aortoarteritis (Takayasu's arteritis). Am Heart J 1991;122:1666-70.

26. Popiołek L, Siga O, Dzieza-Grudnik A, et al. Personality traits and hypertension-mediated organ damage. Psychiatr Pol 2019;53:1003-20.

27. Zhu H, Cao W, Zhao P, et al. TRIF/EGFR signalling mediates angiotensin-II-induced cardiac remodelling in mice. J Mol Endocrinol 2020;65:11-20.

28. Raman VK, Lee YA, Lindpaintner K. The cardiac reninangiotensin-aldosterone system and hypertensive cardiac hypertrophy. Am J Cardiol 1995;76:18D-23D.

29. Izumaru K, Hata J, Nakano T, et al. Reduced Estimated GFR and Cardiac Remodeling: A Population-Based Autopsy Study. Am J Kidney Dis 2019;74:373-81. 